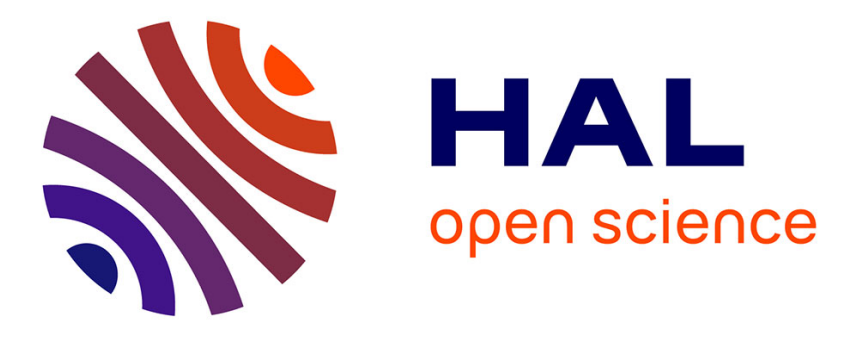

\title{
Epithelial cell adhesion molecule is a prognosis marker for intrahepatic cholangiocarcinoma.
}

\author{
Laurent Sulpice, Michel Rayar, Bruno Turlin, Eveline Boucher, Pascale \\ Bellaud, Mireille Desille, B. Meunier, Bruno Clément, Karim Boudjema,
}

Cédric Coulouarn

\section{To cite this version:}

Laurent Sulpice, Michel Rayar, Bruno Turlin, Eveline Boucher, Pascale Bellaud, et al.. Epithelial cell adhesion molecule is a prognosis marker for intrahepatic cholangiocarcinoma.. Journal of Surgical Research, 2014, 192 (1), pp.117-123. 10.1016/j.jss.2014.05.017 . hal-01069630

\section{HAL Id: hal-01069630 \\ https://hal.science/hal-01069630}

Submitted on 29 Sep 2014

HAL is a multi-disciplinary open access archive for the deposit and dissemination of scientific research documents, whether they are published or not. The documents may come from teaching and research institutions in France or abroad, or from public or private research centers.
L'archive ouverte pluridisciplinaire HAL, est destinée au dépôt et à la diffusion de documents scientifiques de niveau recherche, publiés ou non, émanant des établissements d'enseignement et de recherche français ou étrangers, des laboratoires publics ou privés. 


\section{EpCAM is a prognosis marker for intrahepatic cholangiocarcinoma}

Laurent Sulpice, MD.PhD ${ }^{1-3}$, Michel Rayar, MD ${ }^{2,3}$, Bruno Turlin, MD.PhD ${ }^{1,2,4}$, Eveline Boucher, MD ${ }^{1,2,5}$, Pascale Bellaud ${ }^{1,2}$, Mireille Desille, $\mathrm{PhD}^{1,2}$, Bernard Meunier, MD ${ }^{2,3}$, Bruno Clément, $\mathrm{PhD}^{1,2}$, Karim Boudjema, M.D.Ph.D ${ }^{1-3}$, and Cédric Coulouarn, $\mathrm{PhD}{ }^{1,2}$

${ }^{1}$ Inserm, UMR991, Liver Metabolisms and Cancer, F-35033 Rennes, France;

${ }^{2}$ Université de Rennes 1, F-35043 Rennes, France;

${ }^{3}$ CHU Rennes, Service de Chirurgie Hépatobiliaire et Digestive, F-35033 Rennes, France;

${ }^{4}$ CHU Rennes, Service d'Anatomie et Cytologie Pathologiques, F-35033 Rennes, France;

${ }^{5}$ Centre Régional de Lutte contre le Cancer, F-35042 Rennes, France;

\section{Corresponding author}

Laurent Sulpice, MD.PhD

CHU Rennes, Service de Chirurgie Hépatobiliaire et Digestive, Université de Rennes 1, F-35033 Rennes, France;

Telephone: + 33299284265 Fax: + 33299284129

Email: laurent.sulpice@chu-rennes.fr

Running head: Intrahepatic cholangiocarcinoma and EpCAM

Keywords : Biomarkers ; intrahepatic cholangiocarcinoma; prognosis

\section{Financial support}

This research was supported by Inserm, Université de Rennes1, Association Française pour l'Etude du Foie, Institut National contre le Cancer and Novartis Oncology, France 


\section{Author participation}

LS :1,2,3,4,5 - MR: 2 - BT: 2,3 - EB: 4 - PB and MD :2,3 - BM:5 - BC: 5 - KB and CC : 6

(1) conception and design, (2) analysis and interpretation, (3) data collection, (4) writing the article, (5) critical revision of the article and obtaining funding, (6)approving the final version of the manuscript. 


\section{Abbreviation}

CSC: cancer stem cell

DFS: desease free survival

HCC: hepatocellular carcinoma

HPC: hepatic progenitor cell

HR: hazard ratio

ICC: intrahepatic cholangiocarcinoma

IHC: immunohistochemistry

NFT: non tumor liver fibrous tissue

OS: overall survival

TMA: tissue microarray

TS: tumor stroma 


\section{ABSTRACT}

Background: Recently, we identified a gene signature of intrahepatic choloangiocarcinoma (ICC) stroma and demonstrated its clinical relevance for prognosis. The most up-regulated genes included EpCAM, a cancer stem cells (CSC) biomarker. We hypothesized that CSC biomarkers could predict recurrence of resected ICC.

Methods: Both functional analysis of the stroma signature previously obtained and immunohistochemistry (IHC) of 40 resected ICC were performed. The Relationships between the expression of CSC markers and clinicopathological factors including survival were assessed by univariate and multivariable analyzes.

Results: Gene expression profile of the stroma of ICC highlighted embryonic stem cells signature. IHC on TMA showed at a protein level the increased expression of CSC biomarkers in the stroma of ICC compared to non tumor fibrous liver tissue (NFT). The overexpression of EpCAM in the stroma of ICC is an independent risk factor for overall $(\mathrm{HR}=2.6 ; \mathrm{CI} 95 \%[1.3 ; 5.1] ; \mathrm{p}=0.005)$ and disease free survival $(\mathrm{DFS})(\mathrm{HR}=2.2 ; \mathrm{CI} 95 \%$ [1.2; 4.2]; $\mathrm{p}=0.012$ ). In addition, the overexpression of EpCAM in NFT is closely correlated with a worst DFS $(\mathrm{p}=0.035)$.

Conclusions: Our findings provide new arguments for a potential role of CSC on ICC progression supporting the idea that targeting CSC biomarkers might represent a promise personalized treatment. 


\section{INTRODUCTION}

Intrahepatic cholangiocarcinoma (ICC) is considered to be a therapeutic challenge and a public health issue. Indeed, while ICC incidence is increasing in all western countries[1], complete surgical resection is currently the only available curative treatment. After surgery, the 5-year survival rate of patients with ICC remains low ranging between $25 \%$ and $35 \%$ in most of series $[2,3]$. This poor prognosis is related to the high recurrence rate, especially during the first year after liver resection. Recently, we showed that ICC recurrences occurred in about half of the patients after surgery with curative intent, frequently during the first year and usually in the remnant liver only [4]. This major early recurrence rate as well as the high rate of patients diagnosed with satellite nodes in both lobes, suggests that ICC is a disseminating parenchymal liver disease. The genomics and molecular mechanisms involved in the onset and progression of ICC are poorly documented. Many arguments based on both experimental data and the expression of specific markers indicated that ICC may originate from cancer stems cells (CSC) or hepatic progenitor cells (HPC)[5]. CSC share functional characteristics with normal stem cells including the potential of self-renewal and pluripotency. Several reports argue about the role of CSC in tumor progression, tumor relapse after treatment and resistance to chemotherapy. Accordingly, Sia et al. demonstrated in a genome wide analysis that their subgroup of progressive ICC was associated with a stem-like ICC signature [6]. Recently, by combining laser capture microdissection and gene expression profiling, a gene signature of the tumor stroma (TS) in ICC have been established and associated with a clinical relevance on prognosis[7]. This genomic signature included 1,073 non-redundant genes that significantly discriminate the TS from non-tumor fibrous tissue. Interestingly, the most up-regulated genes in the TS included EpCAM, a well-known marker of CSCs in liver tumors [8]. 
To date, the precise functions of CSC in liver tumors remain poorly understood, particularly due to the lack of accurate cell surface markers that can be isolated on CSC. However, Ma YC et al. showed that the presence of CSCs was associated with a poor histopathologic grade and a worse survival including disease-free survival (DFS) in hepatocellular carcinoma (HCC) [9].

The aim of the study was to determine whether the expression of CSC markers could predict recurrence following surgical resection of ICC. 


\section{METHODS}

\section{Patient Characteristics and Tissue Samples}

Forty patients who underwent liver resection with curative intent for ICC at RennesUniversity hospital between Jan; 1997 and Aug. 2011 were studied. Only mass-forming type ICC, as defined by the Liver Cancer Study Group of Japan, were included and analyzed. Formalin-fixed paraffin-embedded (FFPE) blocks containing tumor and surrounding non tumor liver tissues were retrieved from the archives of the Pathology Institute of Rennes University of. The histology of all tumors was reviewed and confirmed by 2 experienced pathologists and classified according to UICC 7ed. Clinical features were obtained from hospital charts. Data were collected on demographics (age, gender, body mass index), viral status (HBV and $\mathrm{HCV}$ ) and the presence of an underlying liver disease. After resection, the follow-up protocol included a clinical examination and a computed tomography scan every 3 months during 2 years, then every six months thereafter. The end of the follow-up was set between January 1.2013 and Mars 1.2013, or at the time of death. The study protocol fulfilled national laws and regulations and was approved by the local ethics committee.

\section{Data Mining of ICC RNA Profiles}

RNA analysis was made by using the gene expression profiles that we established previously from the micro dissected stroma of human ICC or fibrous tissue in the adjacent non-tumor liver tissue [10]. The full expression dataset was downloaded from the gene expression omnibus (GEO) database (accession number, GSE45001). Gene set enrichment analysis (GSEA) was performed by using the Java-tool developed at the Broad Institute (Cambridge, MA, USA) as previously described [11]. 


\section{Tissue Microarray Construction (TMA)}

To reduce the experimental variations and to standardize the results, immunohistochemistry (IHC) was made by tissue microarray (TMA). TMA design and construction were performed using TMADesigner® software and a Minicore $3{ }^{\circledR}$ tissue Arrayer (Excilone, VICQ France) as previously described [10]. Briefly, after a hematoxylin-eosin staining, 3 representative areas of stroma from each ICC (TS) and of fibrous tissue from portal tracts areas in the surrounding non tumor liver (NFT) were selected by an experienced pathologist (BT) and were punched with a cylinder of $1 \mathrm{~mm}$ diameter before transferred to a TMA block. Thus, each tissue block ( $\mathrm{T}$ and NT) was represented by three independent spots in the TMA. Subsequently, immunohistochemical studies were performed on $4 \mu \mathrm{m}$ tissue sections of TMAs.

\section{Immunostaining for EpCAM, CD44 and CD133}

IHC was performed for CSC markers (EpCAM, CD44 and CD133). As described previously, $4 \mu \mathrm{m}$ tissue sections of the TMAs were deparaffinised and immunostained using an automated Discovery XT immunostaining device (Ventana Medical System, Tuckson, AZ, USA). The following primary monoclonal anti-mouse antibodies were used: EpCAM (1/50, eBiosciences, San Diego, CA), CD44 (1/200, ProMab Biotechnologies, Richmond, CA) and CD133 (1/200, ProMab Biotechnologies, Richmond, CA). Detection was performed using a streptavidin-biotin-peroxidase kit (OmniMap, Biotin-free DAB detection Systems, Ventana Medical System). Staining results were independently scored by experienced pathologist (BT) in a blind manner. Staining intensity in the stroma was scored as follows: negative (0), mild (1), moderate (2), or strong (3). Given that each stromal sample was represented in triplicate, the sum of the three values was calculated to obtain a score ranging from 0 to 9 . This score 
was finally categorized into 4 groups to optimize the statistical analysis and to be expandable for: 0 (score 0-1), 1 (score 2-3), 2 (score 4-7) and 3 (score 8-9).

\section{Statistical Analysis}

Differences in protein expression TS vs. NFT were evaluated by chi-squared test. Relationships between protein expression and clinical parameters were evaluated by chisquared or Fisher's exact probability test for categorical variables and using the analysis of variance for numerical variables. The Kaplan-Meier method was used to estimate the overall (OS) and disease-free survival (DFS) and group differences were analyzed with the log-rank test. Univariate and multivariate Cox regression models for the hazards of OS and DFS mortality were used to evaluate the effect of protein expression. The most suited Cox model was selected using a stepwise regression, selecting variables based on the AIC. $P<0.05$ was considered statistically significant. Statistical analysis was performed with R (version 2.15.1). 


\section{RESULTS}

\section{Clinicopathological Features of Patients}

Clinicopathological features of patients are reported in table 1. The median age at time of surgery was $64.8 \pm 8.8$ years. A full follow-up period was available for all patients with a mean of 38.7 months (ranging from 2 to 118 months). This cohort represents ICC cases encountered in clinical practice, particularly with an even distribution according to the UICC 7ed classification $(37.5 \%, 30 \%, 25 \%$, and $7.5 \%$ for stage I, II, III, and IV respectively). Among the 40 patients, a recurrence occurred in about half of cases during the follow-up period.

\section{The Stroma of ICC Exhibits CSC Gene Signatures}

A supervised analysis of gene expression profiles that we established previously from microdissected ICC tissues demonstrated that the expression of EpCAM was significantly increased in the stroma of ICC compared to fibrous tissue in the NT surrounding tissue ( $\mathrm{p}<0.05$ ). No significant difference was observed for CD44 and CD133 mRNA levels (Figure 1A). Data mining of the full dataset by GSEA demonstrated that gene signatures associated with embryonic stem cells[12] were significantly enriched in the gene expression profiles of the stroma of ICC (normalized enrichment scores, NES >1.5; $\mathrm{p}<0.05$ ). (Figure 1B)

\section{Immunohistochemistry on TMA Confirmed at a Protein Level the Overexpression of CSC} Markers in the Stroma of ICC

Immunostaining on TMA are showed in supplementary figure 1. As reported in figure $1 \mathrm{C}$, a significant overexpression of CSC markers in tumor stroma was found in comparison with 
fibrous areas in the non tumor surrounding liver. The $\mathrm{p}$ values corresponding to the differential expression of EpCAM, CD44 and CD133 were $<0.001,<0.001$ and 0.0284 respectively.

\section{Stromal Expression of EpCAM is an Independent Factor of Prognosis}

Univariate and multivariable analyses of risk factors influencing OS and DFS are reported in Tables 2 and 3. Among the 15 variables assessed by the univariate analysis, an age over 65 years $(p=0.029)$, intrahepatic satellite nodules $(p=0.021)$, CD44 stromal staining $(p<0.001)$ and EpCAM stromal staining $(\mathrm{p}=0.007)$ were significantly associated with OS. Regarding DFS, the univariate analysis showed that tumor size over 50 millimeters $(p=0.023)$, AJCC classification $(\mathrm{p}=0.001)$, intrahepatic satellite nodules $(\mathrm{p}=0.021)$, microvascular invasion ( $\mathrm{p}=0.023)$, perineural infiltration $(\mathrm{p}=0.004)$, capsular disruption $(\mathrm{p}=0.017), \mathrm{CD} 44$ stromal staining $(\mathrm{p}=0.004)$ and EpCAM surrounding non tumor fibrous tissue staining $(\mathrm{p}=0.035)$ were significant.

OS and DFS curves according to EpCAM and CD44 staining are shown in Figures 2 and supplementary figure 2 respectively.

In multivariable analysis, intrahepatic satellite nodules (Hazard ratio [HR] 4.5; confidence interval $[\mathrm{CI}]$ 95\% $[1.5 ; 13.4] ; \mathrm{p}=0.008)$ and EpCAM stromal staining $(\mathrm{HR}=2.6 ; \mathrm{CI} 95 \%$ [1.3; 5.1]; $\mathrm{p}=0.005$ ) remained significant independent risk factors for reduced OS. Independent risk factors of reduced DFS included intrahepatic satellite nodules (HR=3.4; CI 95\% [1.2; 9.7]; $\mathrm{p}=0.026)$, microvascular invasion $(\mathrm{HR}=2.7 ; \mathrm{CI} 95 \%[1.2 ; 6.2] ; \mathrm{p}=0.016)$ and EpCAM stromal staining $(\mathrm{HR}=2.2 ; \mathrm{CI} 95 \%[1.2 ; 4.2] ; \mathrm{p}=0.012)$. 
These results highlighted the correlation between the overexpression of CSC markers and recurrence in ICC.

\section{DISCUSSION}

Intrahepatic cholangiocarcinoma remains characterized by a poor prognosis due to its high rate of recurrence and metastasis after liver resection with curative intent. While several studies have investigated the prognostic value of clinical and histological factors such as age, lymph nodes status, perineural or vascular invasion, margin thickness and the presence of intrahepatic satellite nodes, but few had focused on immunohistological factors. The results of the present study highlight the potential relationship between the expression of CSC biomarkers and the recurrence and poor prognosis of resected ICC. Thus, CSC biomarkers expression was found significantly higher in tumor stroma of ICC than in surrounding fibrous non liver tissue, as previously reported [13]. Univariate analysis revealed that the overexpression of CD44 and EpCAM in tumor stroma was associated with worse OS and DFS. Multivariable analysis confirmed that EpCAM overexpression in tumor stroma was an independent risk factor of poor OS and DFS. Interestingly, the marked staining of EpCAM in the surrounding non-tumor fibrous tissue was also significantly associated with a reduced DFS.

In the early 2000s, the concept of CSC role in tumorigenesis and in cell spreading has been emerged[14]. Experimental evidences suggested that HCC and ICC may derive from common hepatic progenitor cells (HPCs) or oval cells $[5,15,16]$. HPCs are located in the smallest and most peripheral branches of the biliary tree; the ductules and the canals of Hering at the interface between the parenchyma and the portal tract mesenchyme [17]. The activation of HPC compartment can give rise to ductular reactions (DR) as a response to liver damage, 
which is correlated to the degree of inflammation and fibrosis in many chronic liver diseases. Recently, Cai et al. demonstrated that DR/HPCs activation in non-tumor tissue contribute on the risk of recurrence of hepatocholangiocarcinoma (CHC) after curative resection [18]. Similarly, Coulouarn et al. showed by a genome-wide transcriptionnal analysis that this particular of mixed tumor exhibits stem/progenitor features associated with poor prognosis[19].

Several recent reports described a specific niche into the stroma where CSC are located $[20,21]$. The Current evidence revealed that the tumor niche has a pivotal role in controlling cancer homeostasis and progression. In ICC, CSCs are supposed to be generated through the genetic alteration of hepatic stem cells (HSCs), HPCs and probably from cholangiocytes themselves[22]. CSCs are identified through the presence of specific cell surface markers. The most specific currently described CSC biomarkers in cholangiocarcinoma include CD133/prominin-1[23], CD44[24] and EpCAM[25]. Wang et al. demonstrated that $\mathrm{CD}_{4} 4^{+} \mathrm{CD} 24^{+} \mathrm{EpCAM}^{+}$cells isolated from extrahepatic cholangiocarcinoma xenografts in NOD/SCID mice exhibit a higher tumorigenic potential compared with $\mathrm{CD}^{-}{ }^{-} \mathrm{CD}^{-}{ }^{-} \mathrm{EpCAM}^{-}$ cells[26].

The present work provides additional arguments to the hypothesis of the pivotal role of CSC in ICC recurrence. Indeed, we showed a close correlation between DFS and the expression of CSC markers in the tumor stroma of resected ICCs. Overexpression of EpCAM in the surrounding non tumor fibrous tissue was also associated with a reduced DFS, supporting the hypothesis that the presence of CSC in the remnant liver after surgery may contribute on the high rate of early intrahepatic recurrence. In addition, the presence of CSC may explain the ineffectiveness of the current chemotherapy regiment (CT). In fact, conventional CT is efficient on differentiated cells which form the main mass of the tumor, though it fails on 
CSC[21] . Therefore, a combinations of conventional CT and specific treatment targeting CSCs, e.g. through specific cell surface markers might represent a promising strategy to prevent ICC spreading and relapse after surgery. Different approaches could be considered to reverse the drug resistance nature of ICC tumors, including the modulation of the crosstalk between CSC and the tumor niche. A recent study revealed that RNA-based blockage of EpCAM in HSC and HPC led to a decrease in the invasiveness of HCC cell lines [27]. Monoclonal antibodies against EpCAM are now available and were demonstrated to be effective in several solid tumors, namely prostatic carcinoma [28], uterine papillary carcinoma and pancreatic carcinoma[29]. Among the anti-EpCAM antibodies, adecatumumab (MT201) is a safe promising fully human antibody with a long half-life, well tolerated by patients [30].

A limitation of the present work is linked to the small size of the study group, and further studies are needed to confirm the results. Furthermore, EpCAM is a surface biomarker of CSC, but also known to be as a marker widely associated with the epithelial to mesenchymal transition which could partly explain its role in ICC aggressiveness.

In conclusion, the present study suggests a potential role of CSC in the aggressiveness and recurrence of ICC. In addition, we propose EpCAM as a new independent risk factor for poorest survival which can be performed in routine immunohistochemistry. Further studies are mandatory to validate EpCAM as a biomarker for adapted therapies of ICC, including surgical resection of the tumor and the follow-up of patients. In fact, patients with high expression of EpCAM might benefit from an adjuvant treatment using a combination of CT regimens and targeted biotherapy, such as the neutralizing antibodies adecatunumab, which is an appealing strategy and to be explored in the near future.

\section{ACKNOWLEDGEMENTS}


The authors thank the Centre de Ressources Biologiques Santé (Rennes) and the H2P2 hisptopathological platform (Biosit, Rennes) and the National Network of Liver Biobanks. 
References

1. Taylor-Robinson SD, Toledano MB, Arora S, et al.: Increase in mortality rates from intrahepatic cholangiocarcinoma in England and Wales 1968-1998. Gut 2001; 48:816-820.

2. Ercolani G, Vetrone G, Grazi GL, et al.: Intrahepatic cholangiocarcinoma: primary liver resection and aggressive multimodal treatment of recurrence significantly prolong survival. Ann Surg 2010; 252:107-114.

3. Maithel SK, Gamblin TC, Kamel I, et al.: Multidisciplinary approaches to intrahepatic cholangiocarcinoma. Cancer 2013.

4. Sulpice L, Rayar M, Boucher $E$, et al.: Treatment of recurrent intrahepatic cholangiocarcinoma. Br J Surg 2012; 99:1711-1717.

5. Komuta M, Spee B, Vander BS, et al.: Clinicopathological study on cholangiolocellular carcinoma suggesting hepatic progenitor cell origin. Hepatology 2008; 47:1544-1556.

6. Sia D, Hoshida $Y$, Villanueva $A$, et al.: Integrative molecular analysis of intrahepatic cholangiocarcinoma reveals 2 classes that have different outcomes. Gastroenterology 2013; 144:829-840.

7. Sulpice L, Rayar M, Desille M, et al.: Molecular profiling of stroma identifies osteopontin as an independent predictor of poor prognosis in intrahepatic cholangiocarcinoma. Hepatology 2013; 58:1992-2000.

8. de Boer CJ, van Krieken JH, Janssen-van Rhijn CM, Litvinov SV: Expression of Ep-CAM in normal, regenerating, metaplastic, and neoplastic liver. J Pathol 1999; 188:201-206.

9. Ma YC, Yang JY, Yan LN: Relevant markers of cancer stem cells indicate a poor prognosis in hepatocellular carcinoma patients: a meta-analysis. Eur J Gastroenterol Hepatol 2013.

10. Sulpice L, Rayar M, Desille M, et al.: Molecular profiling of stroma identifies Osteopontin as an independent predictor of poor prognosis in intrahepatic cholangiocarcinoma. Hepatology 2013.

11. Coulouarn C, Corlu A, Glaise D, et al.: Hepatocyte-stellate cell cross-talk in the liver engenders a permissive inflammatory microenvironment that drives progression in hepatocellular carcinoma. Cancer Res 2012; 72:2533-2542.

12. Ben-Porath I, Thomson MW, Carey VJ, et al.: An embryonic stem cell-like gene expression signature in poorly differentiated aggressive human tumors. Nat Genet 2008; 40:499-507.

13. Iwahashi $S$, Utsunomiya $T$, Shimada $M$, et al.: High expression of cancer stem cell markers in cholangiolocellular carcinoma. Surg Today 2013; 43:654-660.

14. Reya T, Morrison SJ, Clarke MF, Weissman IL: Stem cells, cancer, and cancer stem cells. Nature 2001; 414:105-111. 
15. Roskams T: Liver stem cells and their implication in hepatocellular and cholangiocarcinoma. Oncogene 2006; 25:3818-3822.

16. Woo HG, Lee JH, Yoon JH, et al.: Identification of a cholangiocarcinoma-like gene expression trait in hepatocellular carcinoma. Cancer Res 2010; 70:3034-3041.

17. Roskams TA, Theise ND, Balabaud C, et al.: Nomenclature of the finer branches of the biliary tree: canals, ductules, and ductular reactions in human livers. Hepatology 2004; 39:17391745.

18. Cai X, Zhai J, Kaplan DE, et al.: Background progenitor activation is associated with recurrence after hepatectomy of combined hepatocellular-cholangiocarcinoma. Hepatology 2012; 56:1804-1816.

19. Coulouarn C, Cavard C, Rubbia-Brandt L, et al.: Combined hepatocellularcholangiocarcinomas exhibit progenitor features and activation of Wnt and TGFbeta signaling pathways. Carcinogenesis 2012.

20. Li L, Xie T: Stem cell niche: structure and function. Annu Rev Cell Dev Biol 2005; 21:605-631.

21. Dong HH, Xiang S, Liang HF, et al.: The niche of hepatic cancer stem cell and cancer recurrence. Med Hypotheses 2013; 80:666-668.

22. Kokuryo $T$, Yokoyama $Y$, Nagino $M$ : Recent advances in cancer stem cell research for cholangiocarcinoma. J Hepatobiliary Pancreat Sci 2012; 19:606-613.

23. Shimada $M$, Sugimoto $K$, Iwahashi $S$, et al.: CD133 expression is a potential prognostic indicator in intrahepatic cholangiocarcinoma. J Gastroenterol 2010; 45:896-902.

24. Wang M, Xiao J, Jiang J, Qin R: CD133 and ALDH may be the molecular markers of cholangiocarcinoma stem cells. Int J Cancer 2011; 128:1996-1997.

25. de Boer CJ, van Krieken JH, Janssen-van Rhijn CM, Litvinov SV: Expression of Ep-CAM in normal, regenerating, metaplastic, and neoplastic liver. J Pathol 1999; 188:201-206.

26. Wang $M$, Xiao J, Shen $M$, et al.: Isolation and characterization of tumorigenic extrahepatic cholangiocarcinoma cells with stem cell-like properties. Int J Cancer 2011; 128:72-81.

27. Yamashita T, Ji J, Budhu A, et al.: EpCAM-positive hepatocellular carcinoma cells are tumorinitiating cells with stem/progenitor cell features. Gastroenterology 2009; 136:1012-1024.

28. Oberneder R, Weckermann D, Ebner B, et al.: A phase I study with adecatumumab, a human antibody directed against epithelial cell adhesion molecule, in hormone refractory prostate cancer patients. Eur J Cancer 2006; 42:2530-2538.

29. Moldenhauer G, Salnikov AV, Luttgau S, et al.: Therapeutic potential of amanitin-conjugated anti-epithelial cell adhesion molecule monoclonal antibody against pancreatic carcinoma. J Natl Cancer Inst 2012; 104:622-634.

30. Schmidt $M$, Ruttinger $D$, Sebastian $M$, et al.: Phase IB study of the EpCAM antibody adecatumumab combined with docetaxel in patients with EpCAM-positive relapsed or refractory advanced-stage breast cancer. Ann Oncol 2012; 23:2306-2313. 


\section{FIGURE LEGENDS}

Figure1. Functional analysis of the ICC stroma signature. (A) Evaluation of relative mRNA level of selected genes demonstrated a significant increased in the expression of EpCAM in the stroma of ICC compared to the surrounding non tumor liver tissue. The p-value was determined using a 2-tailed Student's t-test. (B) Gene set enrichment analysis (GSEA) demonstrated that gene signatures associated with embryonic stem cells were significantly enriched in the gene expression profiles of the stroma of ICC (normalized enrichment scores, NES >1.5; p <0.05). (C) Immunohistological analysis of Epcam, CD44 and CD133 protein expression in TS and NFT.

Figure2. Kaplan-Meier curves and log-rank analysis of survival according to expression of EpCAM. (A) Overall survival according to expression of EpCAM in the stroma of ICC. (B) Disease free survival according to expression of EpCAM in the stroma of ICC. (C) Disease free survival according to expression of EpCAM in the surrounding non tumor fibrous tissue

Supplementary Figure1. Immunohistochemical staining of EpCAM and CD44 in tissue microarray. (A) EpCAM staining score mild in tumor stroma (TS). (B) EpCAM staining score strong in TS. (C) EpCAM staining score mild in non tumor fibrous tissue (NFT). (D) EpCAM staining score moderate in NFT.

Supplementary Figure2. Kaplan-Meier curves and log-rank analysis of survival according to expression of CD44. (A) Overall survival according to expression of CD44 in the stroma of ICC. (B) Disease free survival according to expression of CD44 in the stroma of ICC 
